\title{
Short-range order of germanium selenide glass
}

\author{
A H MOHARRAM* \\ Rabigh College of Science \& Arts, King Abdulaziz University, Rabigh 21911, Saudi Arabia
}

MS received 29 November 2013; revised 14 April 2014

\begin{abstract}
Chalcogenide $\mathrm{Ge}_{20} \mathrm{Se}_{80}$ glass was prepared using the melt-quench technique. The radial distribution function is obtained from $X$-ray diffraction data in the scattering vector interval $0.28 \leq K \leq 6.87 \AA^{-1}$. Reverse Monte Carlo (RMC) simulations are useful to compute the partial pair distribution functions, $g_{i j}(r)$, partial structure fac-

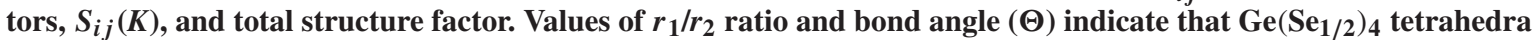
units connected by chains of the chalcogen atoms are present. The partial structure factors have shown that homopolar Ge-Ge and Se-Se bonds are behind the appearance of the first sharp diffraction peak (FSDP) in the total structure factor. Tetrahedral $\mathrm{Ge}\left(\mathrm{Se}_{1 / 2}\right)_{4}$ structural units connected by $\mathrm{Se}-\mathrm{Se}$ chains have been confirmed by the simulated values of the partial coordination numbers and bond angle distributions. Finally, Raman spectra measurements have strongly supported the conclusions obtained either from the calculated Fourier data or from RMC simulations.
\end{abstract}

Keywords. Chalcogenides; X-ray diffraction; short-range order; medium-range order; reverse Monte Carlo simulation.

\section{Introduction}

Chalcogenide glasses present a great potential for application in technological devices, such as optical fibers, memory materials and switching devices, but their use is limited due to several factors. One of them is the difficulty in obtaining information about atomic structures. The structure of chalcogenide glasses in the short-range order (SRO) or intermediate-range order (IRO) is an important and controversial subject. The appearance of the first sharp diffraction peak (FSDP) in the total structure factor indicates the presence of IRO. Germanium selenides have been intensively studied by several methods like X-ray diffraction, ${ }^{1-4}$ neutron diffraction, ${ }^{5,6}$ Raman scattering, ${ }^{7}$ anomalous $\mathrm{X}$-ray scattering ${ }^{8}$ and extended $\mathrm{X}$-ray absorption fine structure. ${ }^{9}$ The structure unit in these glasses is $\mathrm{Ge}\left(\mathrm{Se}_{1 / 2}\right)_{4}$ tetrahedra connected through Se chains.

As mentioned earlier, ${ }^{10}$ the addition of germanium into the polymeric Se matrix produces a cross-linking of selenium chains, mediated by the formation of $\mathrm{Ge}\left(\mathrm{Se}_{1 / 2}\right)_{4}$ tetrahedra. At low doping $(x<15$ at $\%)$, the tetrahedra are sparsely distributed in the background matrix, with rather flexible interconnections. The feeble $\mathrm{Ge}-\mathrm{Ge}$ correlations are inadequate to give any detectable FSDP, as it was previously concluded from the partial pair structure studies on $\mathrm{GeSe}_{2} \cdot{ }^{11}$ When germanium content reaches 15 at $\%$, the amount of Ge atoms becomes sufficient to join some of the tetrahedral pairs by corner sharing. ${ }^{12} \mathrm{X}$-ray diffraction (XRD) of the glassy $\mathrm{Ge}_{x} \mathrm{Se}_{1-x}$ (with $0 \leq x \leq 0.33$ ) systems ${ }^{1,8}$ have

\footnotetext{
*Author for correspondence (mohar200@yahoo.com, amoharram@kau.edu.sa)
}

demonstrated that besides the well-established SRO information, a pre-peak appeared in the total structure factor, $S(K)$, at a scattering vector $K$ of about $1.1 \AA^{-1}$. The pre-peak, being a clear evidence for the existence of the IRO, showed a systematic decrease in intensity and shift towards higher $K$ values with the decrease in Ge concentration. A similar result was observed using the neutron diffraction measurement. ${ }^{5}$ In recent times, ${ }^{13}$ the sorption ability of $\mathrm{Ge}_{20} \mathrm{Se}_{80}$ thin films, applied as active layers of quartz crystal microbalance for $\mathrm{NO}_{2}$ gas sensing, has been investigated. It was found that the introduced gas molecules interact electrostatically with the chalcogen atoms of the host material and initiate some degree of structural changes in it.

Reverse Monte Carlo (RMC) simulation ${ }^{14,15}$ represents, when used carefully, a powerful tool to extract some information of intermediate and extended-range scale in glassy materials. It assembles three-dimensional atomic configurations using the experimental diffraction data implicitly in the simulation. The intimate connection between computational and experimental processes means that the better quality and higher resolution of the experimental data, the more reliable RMC model of a network structure for vitreous materials. RMC method is an inverse problem in which the experimental data are enforced to build atomic configurations that have the desired structural and electronic properties. The main point is to set up a generalized function containing as much information as possible, and then optimize the function for generating configurations toward exact agreement with the experimental data.

The present paper aims to get the structural correlations of the investigated $\mathrm{Ge}_{20} \mathrm{Se}_{80}$ glass either from Fourier transformation or from RMC simulations. It is intended to 
confirm the coincidence between the obtained results of both methods. Finally, Raman spectroscopy analysis could strengthen the obtained conclusions.

\section{Theoretical background}

\subsection{Conventional (Fourier) method}

According to Faber and Ziman, ${ }^{16}$ the total structure factor, $S(K)$, is obtained from the normalized coherent scattered intensity, $I_{\mathrm{c}}(K)$, through

$$
S(K)=\frac{I_{\mathrm{c}}(K)-\left(\left\langle f^{2}(K)\right\rangle-\langle f(K)\rangle^{2}\right)}{\langle f(K)\rangle^{2}},
$$

where $K=4 \pi(\sin \theta / \lambda)$ is the transferred momentum, $\langle f\rangle^{2}=\sum_{i}\left(c_{i} f_{i}\right)^{2}$ and $\left\langle f^{2}\right\rangle=\sum_{i} c_{i} f_{i}^{2}$, where $c_{i}$ is the atomic fraction of element $i$ and $f(K)$ the atomic scattering factor.

Fourier transformation of the $S(K)$ data into real space ${ }^{17}$ gives the reduced distribution function, $G(r)$, as follows:

$$
\begin{aligned}
G(r)= & 4 \pi r\left[\rho(r)-\rho_{\mathrm{o}}\right]=(2 / \pi) \int_{0}^{\infty} K[S(K)-1] \\
& \times M(K) \sin (K r) \mathrm{d} K,
\end{aligned}
$$

where $\rho(r)$ is the local atomic density at a distance $r, \rho_{\mathrm{o}}$ the bulk atomic density and $M(K)$ is called the damping factor. ${ }^{17,18}$ At short distances $(r \leq 2 \AA)$, see Equation (2), $G(r)$ should follow the density line $\left(-4 \pi r \rho_{\mathrm{o}}\right)$ which is used as a quality check of the data. The radial distribution function, defined as the number of atoms lying at distances between $r, r+\mathrm{d} r$ from center of an arbitrary origin atom, is given by

$$
\operatorname{RDF}(r)=4 \pi r^{2} \rho(r)=r G(r)+4 \pi r^{2} \rho_{0} .
$$

The positions of the first and the second peak in the $\operatorname{RDF}(r)$ represent the average values of the first- and second-nearestneighbor distances $r_{1}$ and $r_{2}$, respectively. A knowledge of both distances yields a value for the bond angle $\Theta=$ $2 \sin ^{-1}\left(r_{2} / 2 r_{1}\right) .{ }^{5}$ The area under each peak gives the corresponding coordination number.

\subsection{Reverse Monte Carlo method}

In the structural analysis using Fourier transformations, ${ }^{17,19}$ a modification factor was suggested to reduce the effect of termination data at a finite $K_{\max }$. This factor in turn, while reduces the spurious oscillations, leads to a broadening of the genuine peaks in $g(r)$. The broadening is wide enough to cause an overlap between the first and second peaks, and consequently introduces significant errors in the obtained structural parameters. One of the main difficulties in the study of glasses and other disordered materials is the production of structural models that agree quantitatively with diffraction data. In normal Monte Carlo simulation, an initial structure is allowed to rearrange in such a way that its energy is minimized. The RMC does not need the inter-atomic potentials and the structural configuration is adjusted so as to minimize instead the difference between the calculated diffraction pattern and that measured experimentally. ${ }^{20}$

Three-dimensional arrangement of $\mathrm{N}$ atoms is placed into a cubic cell with periodic boundary conditions. The atomic number density $(\rho)$ should be the same as the experimental value. The positions of the atoms are chosen randomly. The partial pair distribution function ${ }^{15,21}$ can be calculated from the initial configuration by

$$
g_{i j}^{\mathrm{Co}}(r)=\frac{n_{i j}(r)}{4 \pi r^{2} \mathrm{~d} r \rho c_{i}},
$$

where the superscripts $\mathrm{C}$ and o mean 'calculated' and 'old', respectively, $c_{i}$ is the concentration of atoms type $i$ and $n_{i j}(r)$ is the average number of atoms type $j$ located at distance between $r$ and $r+\mathrm{d} r$ from a central atom of type $i$. Fourier transform of $g_{i j}^{\mathrm{Co}}(r)$ to reciprocal space yields the partial static structure factor

$$
S_{i j}^{\mathrm{Co}}(K)=\rho \int_{0}^{\infty} 4 \pi r^{2}\left(g_{i j}^{\mathrm{Co}}(r)-1\right) \frac{\sin K r}{K r} \mathrm{~d} r,
$$

where $K(=4 \pi \sin \theta / \lambda)$ is the momentum transfer. The total structure factor is calculated as follows:

$$
S^{\mathrm{Co}}(K)=\sum_{i, j} c_{i} c_{j} f_{i}(K) f_{j}(K)\left(S_{i j}^{\mathrm{Co}}(K)-1\right),
$$

where $f_{i}(K)$ is the atomic scattering factor of atom type $i$. The difference between the experimental total structure factor, $S^{\mathrm{E}}(K)$, and that calculated from the configuration is given by

$$
\chi_{\mathrm{o}}^{2}=\sum_{i=i}^{m}\left(S^{\mathrm{Co}}\left(K_{i}\right)-S^{\mathrm{E}}\left(K_{i}\right)\right)^{2} / \sigma^{2}\left(K_{i}\right),
$$

where the sum is taken over the $m$ experimental points and $\sigma$ represents the experimental error. One atom moves at random but if it approaches another atom closer than the cut-off distance, the move is rejected. Otherwise, a new atom is chosen with acceptable move. Then, the new values of the partial pair distribution functions, partial structure factors and the total structure factor can be calculated. The new value of $S^{\mathrm{Cn}}(K)$ gives a new difference

$$
\chi_{\mathrm{n}}^{2}=\sum_{i=i}^{m}\left(S^{\mathrm{Cn}}\left(K_{i}\right)-S^{\mathrm{E}}\left(K_{i}\right)\right)^{2} / \sigma^{2}\left(K_{i}\right),
$$

where $\mathrm{n}$ means 'new'. If $\chi_{\mathrm{n}}^{2}<\chi_{\mathrm{o}}^{2}$, the move is accepted and the new configuration becomes the old one. If $\chi_{\mathrm{n}}^{2}>\chi_{\mathrm{o}}^{2}$, it is accepted with probability $\exp \left(-\left(\chi_{n}^{2}-\chi_{0}^{2}\right) / 2\right)$. Otherwise it is rejected. As the number of accepted atom moves increases, $\chi^{2}$ will initially decrease until it reaches an equilibrium value. Thus, the atomic configuration corresponding to the equilibrium should be consistent with the experimental 
total structure factor within the experimental error. From the equilibrium values of the partial pair distribution function, one can calculate the partial coordination number, partial inter-atomic distance and the bond angle distribution.

\section{Experimental}

Bulk $\mathrm{Ge}_{20} \mathrm{Se}_{80}$ chalcogenide was prepared using the melt-quench technique. High-purity $\mathrm{Ge}$ and $\mathrm{Se}$ elements (99.999\%) were, weighed according to their atomic percentages, charged into chemically cleaned silica tube and then sealed under vacuum of $\approx 1.33 \times 10^{-3} \mathrm{~Pa}$. The ampoule was inserted into a furnace where the temperature was raised gradually by rate of $3-4 \mathrm{~K} \mathrm{~min}^{-1}$ up to $1300 \mathrm{~K}$ and kept constant for $24 \mathrm{~h}$. To get homogeneous melt, the ampoule was frequently rocked inside the furnace at the highest temperature. The quenching was made in ice cold water. The glassy state of the quenched alloy was checked using a Philips (PW-1710) X-ray diffractometer. XRD patterns are recorded at scanning speed of $2.4 \mathrm{deg} \mathrm{min}^{-1}$ using $\mathrm{CuK} \alpha$ line $(\lambda=1.5418 \AA)$. The experiment was carried out in the scattering angle range $4^{\circ} \leq 2 \theta \leq 115^{\circ}$ in steps of $0 \cdot 1^{\circ}$, which corresponds to $K$-range $0.284 \leq K \leq 6.874 \AA^{-1}$. Raman spectra was carried out using the $532 \mathrm{~nm}$ line of a diode pumped solid state laser. The scattered light is analyzed with a spectrometer equipped with holographic grating and detected with a Andor Newton CCD camera.

\section{Results and discussion}

As a starting point, the observed X-ray intensities have been corrected through background subtraction followed by absorption and polarization corrections. The corrected X-ray data are used to calculate the total structure factor ${ }^{18}$ as a function of the scattering vector $(K=4 \pi \sin \theta / \lambda)$. As shown in figure 1, a FSDP which is commonly observed in covalently bonded materials implies the presence of IRO caused by connecting some of the structural units. In order to determine the position and intensity of the pre-peaks, the $S(K)$ spectra have been analyzed using two pseudo-Voigt functions ${ }^{1}$ for the pre- and first-peaks. The pseudo-Voigt function is linear summation of Lorentzian and Gaussian components [c.Gauss. $+(1-c)$ Lorent.], where the Gaussian fraction has a value in the range $0 \leq c \leq 1.0$. The values of the characteristic length $\left(R=2 \pi / K_{\mathrm{o}}\right.$, where $K_{\mathrm{o}}$ is the peak position) and the coherence length $(L=2 \pi / \Delta K$, where $\Delta K$ is the halfwidth at half-maximum of the peak), as calculated from the well-resolved FSDP following the procedures published by, Johnson et $a l^{22}$ are $5.45 \pm 0.05$ and $17.44 \pm 0.52 \AA$, respectively.

The reduced distribution function, $G(r)$, calculated after one damping correction followed by several Kaplow iterations, ${ }^{23}$ is shown in figure 2 . At small values of $r \leq 1.5 \AA$, see Equation (2), $G(r)$ function shows a straight line with slope is equal to $-4 \pi \rho_{\mathrm{o}}$. The bulk density obtained from the straight portion of $G(r)$, equals $(3.85 \pm 0.04) \times 10^{-2}$ atom $\AA^{-3}$.

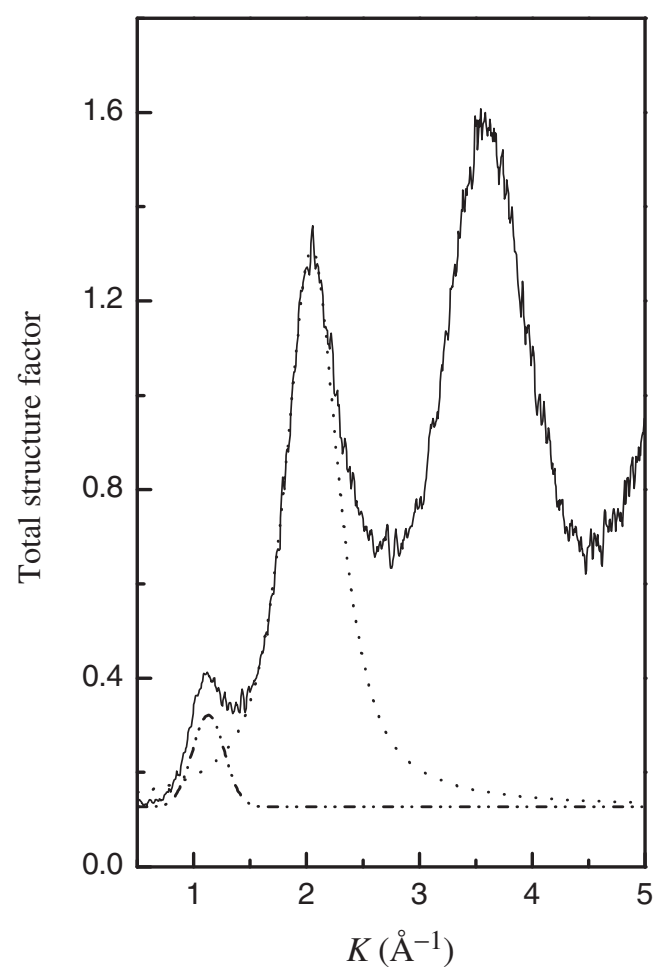

Figure 1. The total structure factor of the $\mathrm{Ge}_{20} \mathrm{Se}_{80}$ glass as a function of the scattering vector $(K)$. The spectra was analyzed using two pseudo-Voigt functions for the pre- and first-peaks.

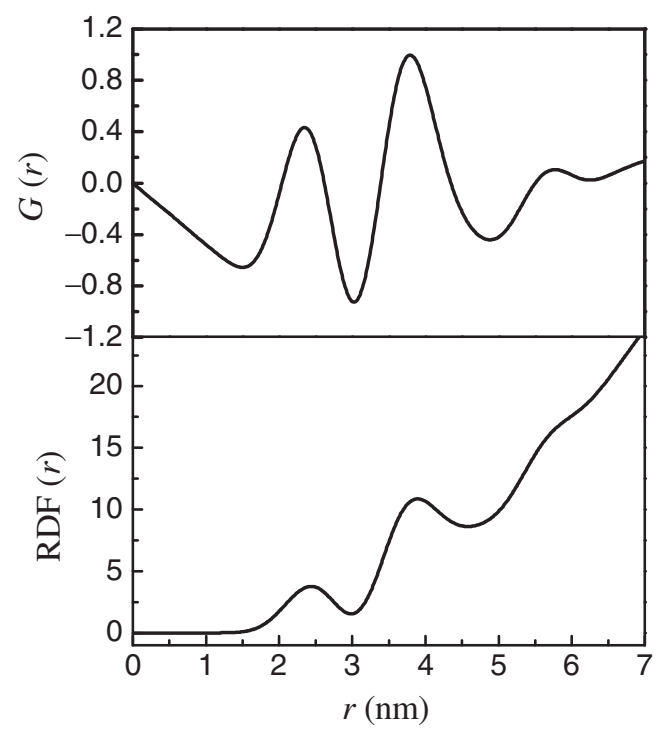

Figure 2. The reduced distribution function, $G(r)$, and the radial distribution function, $\mathrm{RDF}(r), v s . r$ of the investigated $\mathrm{Ge}_{20} \mathrm{Se}_{80}$ glass.

The bulk density can also be calculated from the relation $\rho_{\mathrm{o}}=N_{\mathrm{A}} \sum_{i} x_{i} d_{i} / A_{i}$, where $d_{i}, A_{i}$ and $N_{\mathrm{A}}$ are the density, atomic weight of the element $i$ and Avogadro number, respectively. ${ }^{24}$ The excellent coincidence between the former value of the bulk density and the calculated one 


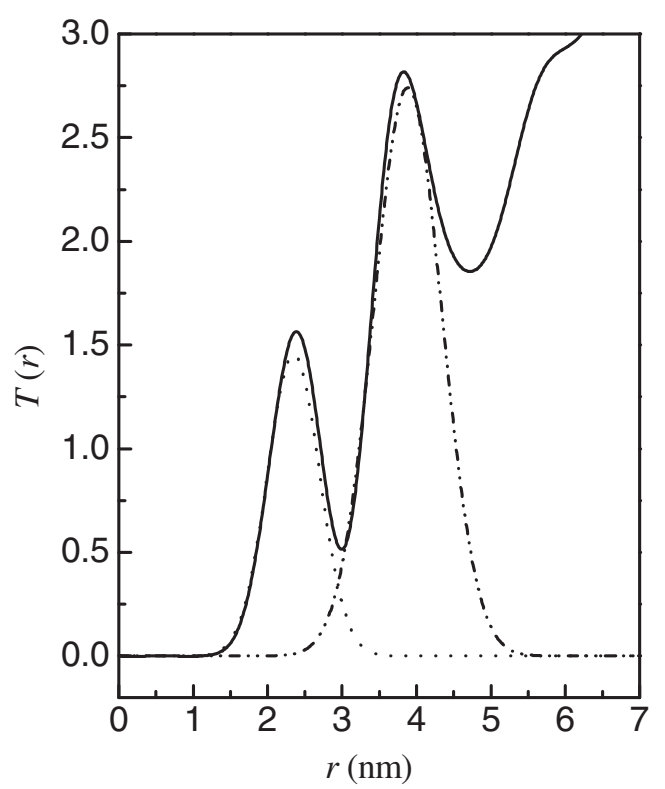

Figure 3. The total distribution function, $T(r), v s . r$ of the studied $\mathrm{Ge}_{20} \mathrm{Se}_{80}$ glass.

$\left(\rho_{\mathrm{o}}=3.8505 \times 10^{-2}\right.$ atom $\left.\AA^{-3}\right)$ confirms the high quality of the present glass. The radial distribution function, $\operatorname{RDF}(r)$, is shown also in figure 2. At high values of $r$, the curves should fit the parabola, $\operatorname{RDF}(r)=4 \pi r^{2} \rho_{\mathrm{o}}$. The broadening appeared in the $\operatorname{RDF}(r)$ peaks is expected to cause significant errors in determining the SRO parameters. So instead of $\mathrm{RDF}(r)$, the total distribution function, $T(r)=\mathrm{RDF}(r) / r$, is commonly used to get the SRO parameters. ${ }^{18}$ The sharpness of the $T(r)$ peaks shown in figure 3 is much better than that appeared in the $\mathrm{RDF}(r)$ curve. Gaussian fit of $T(r)$ curve shown in figure 3 has resulted in two well-resolved peaks, where the positions of the first and second peaks are $r_{1}=2.38 \AA$ and $r_{2}=3.82 \AA$, respectively. These values are in good agreement with those previously obtained by Rao et al. ${ }^{5}$

The starting point in RMC simulation ${ }^{21}$ is to randomly generate the configuration distribution of $N=4000$ atoms inside a cubic box. The length of the cubic configuration is $23.6 \AA$. According to their atomic percentages, the numbers of $\mathrm{Ge}$ and Se atoms inside the cube are 800 and 3200 atoms, respectively. RMC simulation runs for $20 \mathrm{~h}$ using the total structure factor fit under the coordination constraints and a minimum approach distance of $1.96 \AA$ for any atoms pair. When $\chi^{2}$ oscillates around an equilibrium value, a threedimensional molecular image of a disordered structure can be obtained. In order to get an accurate image, the average of five simulation trials was taken. Figure 4 shows the partial pair distribution $g_{\mathrm{Ge}-\mathrm{Ge}}(r), g_{\mathrm{Ge}-\mathrm{Se}}(r), g_{\mathrm{Se}-\mathrm{Se}}(r)$ functions. In fact, most of the important structural parameters such as the coordination number, inter-atomic distance and bond angle distribution can be obtained from the partial pair distribution functions. In the first coordination sphere, the near-zero value of $g_{\mathrm{Ge}-\mathrm{Ge}}(r)$ indicates that only homopolar $\mathrm{Se}-\mathrm{Se}$ bonds exist in addition to heteropolar $\mathrm{Ge}-\mathrm{Se}$ bonds

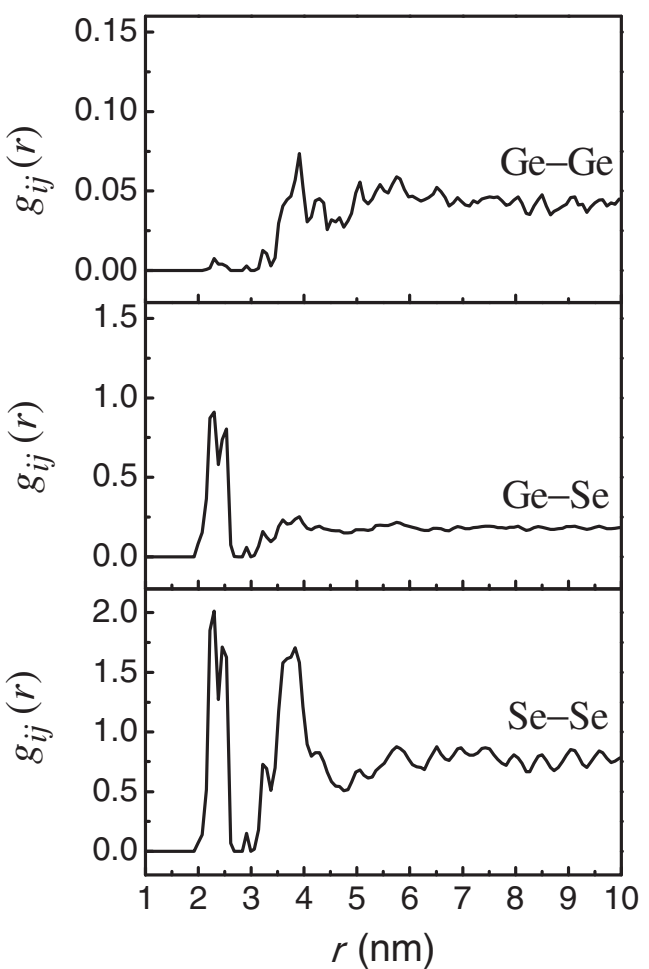

Figure 4. The partial pair distribution $g_{\mathrm{Ge}-\mathrm{Ge}}(r), g_{\mathrm{Ge}-\mathrm{Se}}(r)$, $g_{\mathrm{Se}-\mathrm{Se}}(r)$ functions vs. $r$ of the investigated $\mathrm{Ge}_{20} \mathrm{Se}_{80}$ glass.

Table 1. The partial coordination numbers of the $\mathrm{Ge}_{20} \mathrm{Se}_{80}$ glass as obtained from: RMC, CONM and from other references. ${ }^{5,11}$

\begin{tabular}{|c|c|c|c|c|}
\hline & $\mathrm{Ge}-\mathrm{Ge}$ & $\mathrm{Ge}-\mathrm{Se}$ & $\mathrm{Se}-\mathrm{Ge}$ & $\mathrm{Se}-\mathrm{Se}$ \\
\hline Present & 0.03 & 4.04 & 1.01 & 2.26 \\
\hline CONM & 0 & 4 & 1 & 1 \\
\hline Rao et $a l^{5}$ & 0.22 & 3.61 & 0.9 & 1.71 \\
\hline Salmon and Petri ${ }^{11}$ & 0.22 & 3.61 & 0.9 & 1.71 \\
\hline
\end{tabular}

The average $\mathrm{Ge}-\mathrm{Ge}, \mathrm{Ge}-\mathrm{Se}$ and $\mathrm{Se}-\mathrm{Se}$ bond lengths are, as obtained from the refined RMC model, $2.52 \pm 0.065$, $2.43 \pm 0.065$ and $2.47 \pm 0.065 \AA$, respectively. The average partial coordination numbers, listed in table 1 , are close to some extent to those reported by other references. ${ }^{6,7}$ Based on the chemical order network model (CONM), the partial coordination numbers are also calculated and listed in table 1 . The presence of Se-Se bridges between the tetrahedral units is a possible reason for high partial Se-Se coordination number as compared with that reported previously or computed from the chemically ordered network model. Pan et $a l^{25}$ have characterized the structural units of the $\mathrm{Ge}-\mathrm{Se}$ films prepared by pulsed laser deposition using Raman spectroscopy. They concluded that in addition to the basic structural units of edge-sharing $\mathrm{GeSe}_{4 / 2}$ tetrahedra, there are Se-Se homopolar bonds in Se-rich $\mathrm{GeSe}_{4}$ films.

Bond angle distribution functions, $\Theta(\theta)$, are obtained from the final configuration of the investigated glass using triplets program. ${ }^{7}$ In which, the bond angle for any reference 


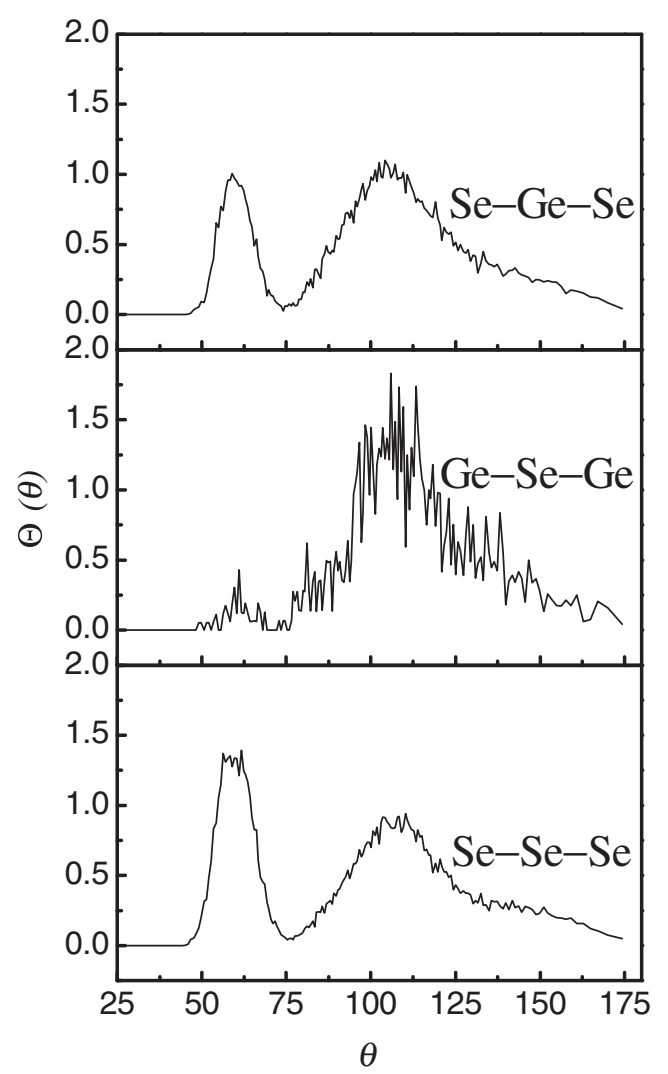

Figure 5. Bond angle distribution functions, $\Theta(\theta)$, obtained from RMC simulations.

atom can be calculated from the Cartesian coordinates of the final positions of the surrounding atoms. The $\Theta(\theta)$ functions of the $\mathrm{Ge}_{20} \mathrm{Se}_{80}$ glass are shown in figure 5. $\Theta_{\mathrm{Se}-\mathrm{Ge}-\mathrm{Se}}(\theta)$ function presents a main peak around $104.2^{\circ}$, which is close to the ideal tetrahedral angle of $109^{\circ}$. A small peak appears at $60^{\circ}$ can be attributed to the existence of what we can call it as wrong (homo) bonds. During the melt-quench process, the freeze of $\mathrm{Ge}$ atoms in some corner positions of the tetrahedral units instead of Se atoms could be responsible for the above small peak. A reverse behavior is given by $\Theta_{\mathrm{Se}-\mathrm{Se}-\mathrm{Se}}(\theta)$ function, where a main peak is located at $60^{\circ}$ and a small one at $104.2^{\circ}$. Selenium atoms occupying face of a perfect tetrahedron should exhibit internal angles of $60^{\circ}$. The presence of a $\Theta_{\mathrm{Se}-\mathrm{Se}-\mathrm{Se}}(\theta)$ peak at $104.2^{\circ}$ can be attributed to intertetrahedral units. The above bond angle distributions have strongly confirmed that the distorted tetrahedral units in addition to the ideal units are formed inside the cube. These structural units seem to be connected by Se-Se bridges, forming small chains and rings, as previously reported. ${ }^{24}$ In the same figure, $\Theta_{\mathrm{Ge}-\mathrm{Ge}-\mathrm{Ge}}(\theta)$ function shows a broad distribution from $50^{\circ}$ to $150^{\circ}$, which indicates that pairs of tetrahedral units can be connected either by corner or edge share.

Fourier transformation of the partial pair distribution gives the corresponding partial scattering factor. The dependence of the partial scattering factors on the scattering vector $(K)$ are shown in figure 6 . The partial $S(K)$ are important especially in regions where the FSDP is located. Because of its insignificant contribution to the pre-peak, $S_{\mathrm{Ge}-\mathrm{Se}}(K)$

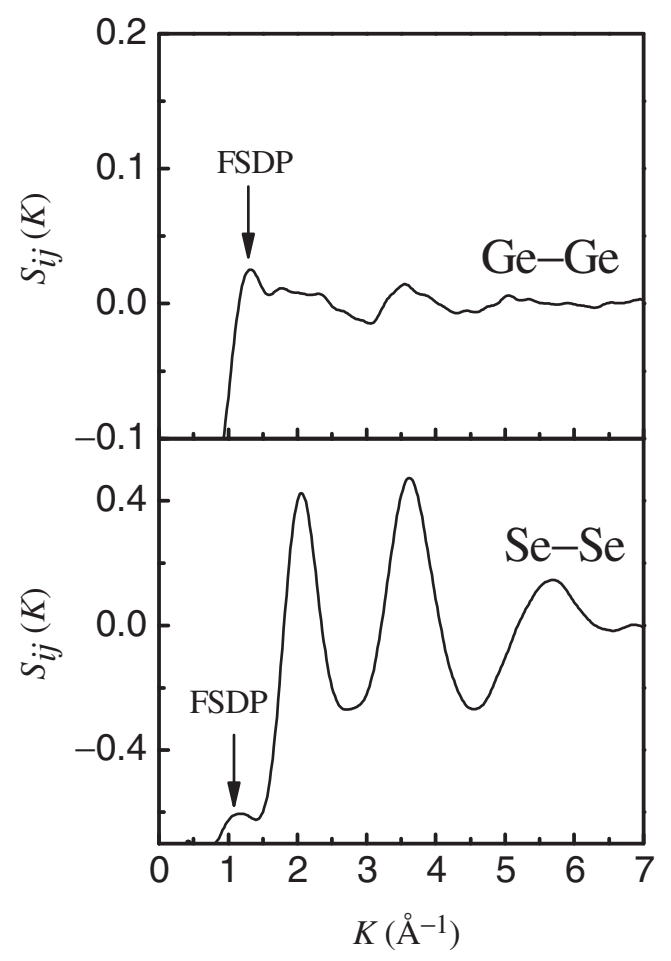

Figure 6. The partial scattering factors, $S_{i j}(K)$, for the studied $\mathrm{Ge}_{20} \mathrm{Se}_{80}$ glass.

function is not shown. It was previously mentioned ${ }^{26}$ that the pre-peak is originated by $\mathrm{Ge}-\mathrm{Ge}$ correlation, which bridging $\mathrm{GeSe}_{4 / 2}$ tetrahedra. Recently, Machado et $a l^{7}$ have used the RMC simulations and noticed the existence of large number of Se-Se pairs in the first coordination shell suggesting that the tetrahedral units are linked by $\mathrm{Se}-\mathrm{Se}$ bridges. The FSDP appeared in the partial $S_{\mathrm{Ge}-\mathrm{Ge}}(K)$ and $S_{\mathrm{Se}-\mathrm{Se}}(K)$ functions indicates that the intermediate range order is not only attributed to $\mathrm{Ge}-\mathrm{Ge}$ bonds but also to the presence of $\mathrm{Se}-\mathrm{Se}$ bonds. Summation of the partial scattering factors gives the total RMC scattering factor as a function of the scattering vector. Figure 7 shows a very good coincidence between RMC simulation and the experimental scattering factor to the point no one can distinguish between their values. In the above figure, the RMC curve is shifted downward by 0.05 in order to differentiate between their values.

Based on the present IRO and SRO parameters, some conclusions about the structural correlations inside the glass matrix are assumed. To confirm such conclusions, Raman spectra measurements of the investigated glass is made and shown in figure 8. Two broad as well as one side (shoulder) peaks have appeared. One of the main peaks located at $267 \mathrm{~cm}^{-1}$ is related to Se-Se pairs. The second main peak located at $200 \mathrm{~cm}$ is assigned to the stretching mode of the corner-sharing (CS) $\mathrm{GeSe}_{4 / 2}$ tetrahedra. The latter peak is accompanied by a shoulder at $215 \mathrm{~cm}^{-1}$, which rises from the vibrations of $\mathrm{Se}$ atoms in the four member rings composed of two edge-sharing (ES) tetrahedra. ${ }^{27}$ The intensities difference between the peak and its shoulder clarify that 


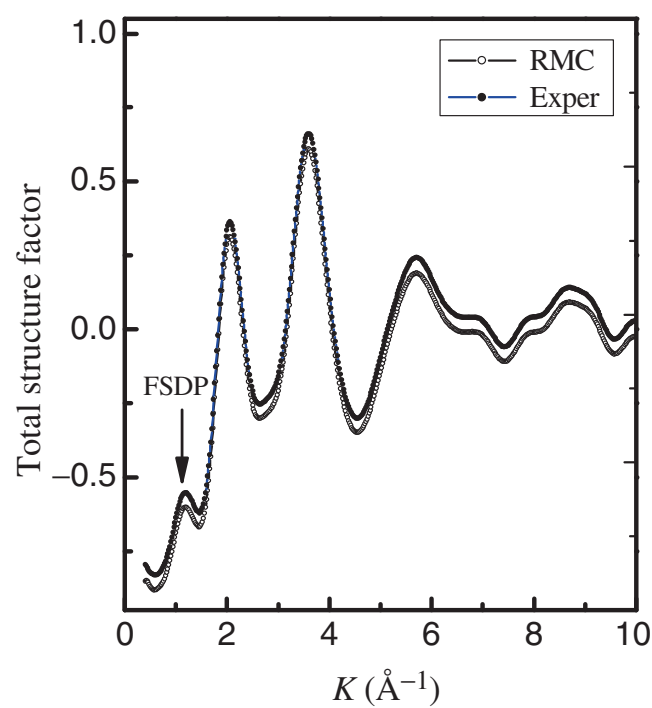

Figure 7. Experimental scattering factor together with RMC simulation of the studied $\mathrm{Ge}_{20} \mathrm{Se}_{80}$ glass. RMC data are shifted downward $(-0.05)$ to clarify the coincidence.

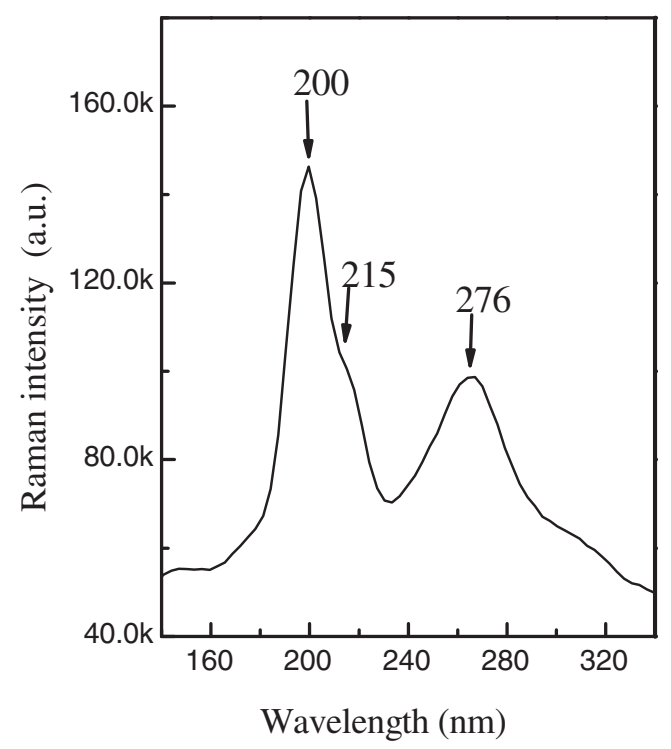

Figure 8. Raman spectrum vs. Raman frequency of the investigated $\mathrm{Ge}_{20} \mathrm{Se}_{80}$ glass.

the studied glass has a lot of CS tetrahedra and few ES tetrahedra.

\section{Conclusions}

The FSDP appeared in the structure factor curve implies the presence of IRO caused by connecting some of the structural units. The values of $r_{1} / r_{2}$ ratio and the corresponding bond angle $(\Theta)$, obtained from the conventional (Fourier) method, indicate that the structural units inside the present alloy are $\mathrm{Ge}\left(\mathrm{Se}_{1 / 2}\right)_{4}$ tetrahedra connected by chains of the chalcogen atoms. RMC simulations of the X-ray scattering data are useful to compute the partial pair distribution functions, $g_{i j}(r)$, the partial structure factors, $S_{i j}(K)$, and consequently the total structure factor. The partial structure factors have shown that not only the homopolar $\mathrm{Ge}-\mathrm{Ge}$ bonds, but also $\mathrm{Se}-\mathrm{Se}$ bonds are behind the appearance of the FSDP in the total structure factor. The presence of the tetrahedral $\mathrm{Ge}\left(\mathrm{Se}_{1 / 2}\right)_{4}$ structural units which connected by $\mathrm{Se}-\mathrm{Se}$ chains have been confirmed by the simulated values of the partial coordination numbers and the bond angle distributions. Finally, Raman spectra measurements have strongly supported the conclusions obtained either from the calculated Fourier data or from RMC simulations.

\section{Acknowledgement}

This work was supported by the Deanship of Scientific Research (DSR), King Abdulaziz University, Jeddah, under Grant No. (662-4-D1432). The author, therefore, acknowledges with thanks DSR technical and financial support.

\section{References}

1. Wang Y, Ohata E, Hosokawa S, Sakurai M and Matsubara E 2004 J. Non-Cryst. Solids 33754

2. Sharma D, Sampath S, Lalla N P and Awasthi A M 2005 Physica B 357290

3. Inam F, Shatnawi M T, Tafen D, Billinge S J L, Chen P and Drabold D A 2007 J. Phys.: Condens. Matter. 19455206

4. Chen G, Inam F and Drabold D A 2010 Appl. Phys. Lett. 97131901

5. Rao N R, Krishna P S R, Basu S, Dasannacharya B A, Sangunni K S and Gopal E S R 1998 J. Non-Cryst. Solids 240221

6. Tafen D and Drabold D A 2005 Phys. Rev. B 7154206

7. Machado K D, de Lima J C, Campos C E M, Gasperini A A M, de Souza S M, Maurmann C E, Grandi T A and Pizani P S 2005 Solid State Commun. 133411

8. Hosokawa S, Wang Y, Sakurai M, Bérar J F, Pilgrim W C and Murase K 2003 Nucl. Instrum. Methods Phys. Res. B199 165

9. Gulbrandsen E, Johnsen H B, Endregaard M, Grande T and Stølen S 1999 J. Solid State Chem. 145253

10. Susman S, Price D L, Volin K J, Dejus R J and Montague D G 1988 J. Non-Cryst. Solids 10626

11. Salmon P S and Petri I 2003 J. Phys.: Condens. Matter. 15 S1509

12. Warren B E, Krutter H and Morningstar O 1936 J. Am. Ceram. Soc. 19202

13. Chen P, Mitkova M, Tenne D A, Wolf K, Georgieva V and Vergov L 2012 Thin Solid Films 525141

14. McGreevy R L and Pusztai L 1988 Mol. Simul. 1359

15. McGreevy R L 2001 J. Phys.: Condens. Matter. 13 R877

16. Faber T E and Ziman J M 1965 Philos. Mag. 11153

17. Elliott S R 1990 Physics of amorphous materials, 2nd ed (New York: Longman)

18. Moharram A H and Abdel-Basit A M 2005 Physica B 358279 
19. Szczygielska A, Burian A, Dore J C, Honkimaki V and Duber S 2004 J. Alloys Compd. 362307

20. Keen D A and McGreevy R L 1990 Nature 344423

21. http://wwwisis2.isis.rl.ac.uk/rmc

22. Johnson R W, Price D L, Susman S, Arai M, Morrison T I and Shenoy G K 1986 J. Non-Cryst. Solids $\mathbf{8 3} 251$

23. Kaplow R, Strong S L and Averbach B L 1965 Phys. Rev. 138 A1336
24. Machado M D, de Lima J C, Campos C E M, Grandi T A and Pizani P S 2004 J. Chem. Phys. 120329

25. Pan R K, Tao H Z, Wang J Z, Wang J Y, Chu H F, Zhang T J, Wang D F and Zhao X J 2013 Optik 1244943

26. Fuoss P H, Eisenberger P, Warburton W K and Bienestock A 1981 Phys. Rev. Lett. 461537

27. Dwivedi P K, Tripathi S K, Pradhan A, Kulkarni V N and Agarwal S C 2000 J. Non-Cryst. Solids 266924 